\title{
Effect of mechanical properties of hydrogel nanoparticles on macrophage cell uptake $\dagger$
}

\author{
Xavier Banquy, ${ }^{a}$ Fernando Suarez, ${ }^{b}$ Anteneh Argaw,${ }^{c}$ Jean-Michel Rabanel, ${ }^{a}$ Peter Grutter, ${ }^{b}$ \\ Jean-François Bouchard, ${ }^{c}$ Patrice Hildgen ${ }^{a b}$ and Suzanne Giasson ${ }^{* a d e f}$
}

\author{
Received 2nd December 2008, Accepted 30th June 2009 \\ First published as an Advance Article on the web 3rd August 2009 \\ DOI: $10.1039 / b 821583 a$
}

\begin{abstract}
Uptake and intracellular trafficking of hydrogel nanoparticles (NPs) of $N, N$-diethyl acrylamide and 2-hydroxyethyl methacrylate crosslinked with $N, N^{\prime}$-methylene-bis-acrylamide were studied with a RAW 264.7 murine macrophage cell line. Results show that the uptake rate, the mechanism of internalization and the concentration of internalized NPs are correlated to the NP Young modulus. Soft NPs are found to be internalized preferentially via macropinocytosis while the uptake of stiff NPs is mediated by a clathrin-dependent mechanism. NPs with an intermediate Young modulus exhibit multiple uptake mechanisms. The accumulation rate of the NPs into lysosomal compartments of the cell is also dependent on the NP elasticity. Our results suggest that control over the mechanical properties of hydrogel NPs can be used to tailor the cellular uptake mechanism and kinetics of drug delivery.
\end{abstract}

\section{Introduction}

Different drug-carrier systems, such as liposomes, polymeric micelles, polymer conjugates and polymeric nanoparticles have been investigated for intracellular delivery of therapeutics. Nanoparticles (NPs) based on crosslinked water-soluble polymers forming hydrogels have shown to be very good candidates for drug carriers ${ }^{1}$ because of their low cytotoxicity ${ }^{2}$ and their tunable properties such as swelling and mechanical properties which can be controlled by the crosslinking density. Moreover, hydrogel NPs are relatively stable against biodegradation, ${ }^{3,4}$ show excellent transfection capabilities, ${ }^{5}$ promote uptake by a large number of cell lines, ${ }^{2}$ and can also be used as intracellular nanosensors. ${ }^{6-8}$

The efficiency of therapeutic carriers for intracellular delivery largely depends on their interactions with the cell membrane and the uptake mechanism. Most NPs are found to be internalized into cells by means of endocytic mechanisms. ${ }^{9}$ The interactions of carriers with cell membranes can be made specific by using ligands that can bind to their receptors on cell membranes. This biorecognition interaction usually increases the intracellular internalization. ${ }^{9}$

${ }^{a}$ Faculty of Pharmacy, Université de Montréal, C.P. 6128, succursale Centre-Ville, Montréal, Québec, H3C 3J7, Canada

${ }^{b}$ Department of Physics, McGill University, 3600 University Street, Montréal, Québec, H3A 2T8, Canada

'Ecole d'Optométrie, Université de Montréal, C.P. 6128, succursale Centre-Ville, Montréal, Québec, H3C 3J7, Canada

${ }^{d}$ Groupe de Recherche Universitaire sur le Médicament de l'Université de Montréal, C.P. 6128, succursale Centre-Ville, Montréal, Québec, H3C 3J7, Canada

${ }^{e}$ Department of Chemistry, Université de Montréal, C.P. 6128, succursale Centre-Ville, Montréal, Québec, H3C 3J7, Canada. E-mail: Suzanne. giasson@umontreal.ca; Fax: +01 514340 5290; Tel: +01 5143405175

${ }^{f}$ Center for Self-Assembled Chemical Structures, McGill University, 801 Sherbrooke St. West, Montréal, Québec, H3A 2K6, Canada

$\dagger$ Electronic supplementary information (ESI) available: Cytotoxicity data for the NPs by LDH and MTT assays. See DOI: 10.1039/b821583a
The physicochemical parameters (i.e. surface charge, NP size and substrate elasticity) of polymer-based carriers have been shown to influence the interaction with cells or the intracellular fate of the carriers. For example, the surface charge of polyamidoamine dendrimers was found to be a determinant factor in their intracellular trafficking. ${ }^{5}$ Anionic dendrimers were found to be internalized via caveolae-mediated endocytosis while neutral and cationic dendrimers were mostly internalized by non-clathrin and non-caveolae mediated endocytosis mechanisms in A549 cells. ${ }^{5}$ The surface charge of poly-D,L-lactic acid (PLA) NPs has been shown to strongly affect the intracellular fate in MadinDarby canine kidney (MDCK) cells but to a less extent the entry mechanism. ${ }^{10}$ Both cationic and anionic PLA NPs were found to be mainly internalized by a clathrin-dependent mechanism. Only anionic NPs were found in the lysosomal compartments while significant amounts of cationic NPs were found to be exocytosed. Particle size has also been shown to affect the uptake mechanism and the intracellular fate. ${ }^{11}$ Small polystyrene particles (nanometre size) were found to exhibit higher intracellular uptake compared to microspheres. Moreover, the small particles were found to be internalized by B16F10 murine melanoma cells almost exclusively via a clathrin-dependent pathway and their intracellular trafficking was found to be microtubule dependent. Larger particles were found to be internalized via a caveolaerelated mechanism and systematically exhibited slower kinetics to reach lysosomal compartments. It was recently reported that polystyrene NPs of $24 \mathrm{~nm}$ diameter can be internalized via a nonclathrin and non-caveolae dependent mechanism. It was shown that these NPs accumulate in non-digestive vesicles close to the perinuclear membrane. These findings may open new routes to the efficient delivery of sensitive molecules like DNA or proteins. ${ }^{\mathbf{1 2}}$

The elasticity of the cell substrate was recently found to have a significant effect on the uptake of polyplexes by murine MC3T3-E1 preosteoblasts. ${ }^{13}$ An increase in substrate elasticity led to an increase in the NP uptake which was correlated with enhanced cell proliferation and survival. This study suggests that 
the mechanical properties of the cell-surface (or cell-particle) interface may influence the cellular uptake and intracellular trafficking of drug carriers. In the present study, we investigated the effect of the mechanical properties of hydrogel NPs, more specifically their elasticity, on the cellular uptake and intracellular fate in murine macrophage cells. Macrophage cells represent a good model system for the evaluation of the cytotoxicity of nanoparticulate vectors and for the investigation of treatments against pathologies related to bacterial invasion as most bacteria and pathogens are sheltered from immune defense system in host macrophages. The mechanical properties of the NPs were controlled by varying the concentration of the crosslinking agent used for the NP synthesis and measured using atomic force microscopy (AFM). The NP elasticity was quantified by the Young modulus. The uptake mechanisms of the different NPs were identified using different inhibitors of known entry routes. The intracellular localization of the NPs was assessed by fluorescence microscopy. Colocalization assays with endosomal and lysosomal markers allowed identification of the intracellular pathways pursued by the NPs after their internalization.

\section{Experimental}

\section{Materials}

$N, N$-Diethyl acrylamide (DEA) was synthesized as previously described.${ }^{14}$ Unless stated, all chemical products were purchased from Sigma-Aldrich (Oakville, ON, Canada). Solvents used were analytical grade and all other chemicals were commercially available reagent grade. 2-Hydroxyethyl methacrylate (HEMA) was vacuum distilled prior to use. $N, N^{\prime}$-Methylene-bis-acrylamide (BIS) was recrystallized twice from hexane. Sodium dodecyl sulfate (SDS) was crystallized from pure ethanol. Amonium persulfate (APS), rhodamine B, 1-ethyl-3-(3-dimethylaminopropyl)carbodiimide hydrochloride (EDC) and $N$-hydroxysuccinimide (NHS) were used as received. Water was obtained from a Milli-Q ${ }^{\circledR}$ Gradient System from Millipore equipped with a Quantum ${ }^{\mathrm{TM}}$ cartridge.

\section{Nanoparticle synthesis}

The NPs were synthesized as previously reported. ${ }^{14}$ The monomers (DEA $90 \mathrm{~mol} \%$ and HEMA $10 \mathrm{~mol} \%$, total amount of $\left.5.3 \times 10^{-3} \mathrm{~mol}\right)$, BIS and SDS $(0.040 \mathrm{~g})$ were added to $200 \mathrm{~mL}$ of Milli- $\mathrm{Q}^{\circledR}$ water under stirring followed by nitrogen purging for $30 \mathrm{~min}$ at $70^{\circ} \mathrm{C}$. An aqueous solution of APS $(0.120 \mathrm{~g}$ in $15 \mathrm{~mL})$ was subsequently added to the reacting medium. The reaction was allowed to proceed for $4 \mathrm{~h}$ and then cooled at room temperature before filtering the milky suspension through $2.0 \mu \mathrm{m}$ Millipore Isopore ${ }^{\circledR}$ membrane filters. The filtrate was centrifuged for $15 \mathrm{~min}$ at $15000 \mathrm{rpm}$. The polymer NPs were collected and purified by dialysis for 1 week against Milli- $Q^{\circledR}$ water. Four different batches of NPs were prepared using four different concentrations of BIS. The NPs used in this study are known to be thermosensitive with a lower critical temperature (LCT) value ranging from 23 to $28{ }^{\circ} \mathrm{C}$ depending on the monomer concentration. ${ }^{14}$ All nanoparticles were labeled with rhodamine B using standard EDC/NHS coupling reactions. After labeling, NPs were centrifuged for $15 \mathrm{~min}$ at $15000 \mathrm{rpm}$ and the supernatant was replaced by Milli- $\mathrm{Q}^{\circledR}$ water. The polymer NPs were collected and purified by dialysis in Milli- ${ }^{\circledR}$ water for 1 week.

\section{Nanoparticle characterization}

Particle size and zeta-potential measurements, and surface topography. NP suspensions were prepared at a final concentration of $0.1 \mathrm{mg} \mathrm{mL}^{-1}$ in filtered phosphate buffer saline (PBS) and the particle size distribution was measured using dynamic light scattering with a Nano-ZS Zetasizer (Malvern Instrument Ltd., Malvern, Worcestershire, UK) at $37^{\circ} \mathrm{C}$ in back-scattering mode. The zeta potential of the NP suspensions was measured in triplicate at $37{ }^{\circ} \mathrm{C}$ using the Zetasizer. Surface imaging was carried out in tapping mode using a multimode AFM equipped with a NanoScope IIIa controller (Digital Instruments, Santa Barbara, CA). The NPs were deposited by spreading a $20 \mu \mathrm{L}$ suspension droplet on a freshly cleaved mica surface and stored in $30 \%$ relative humidity $(\mathrm{RH})$ atmosphere $2 \mathrm{~h}$ prior to AFM analysis. All images were obtained in air and at room temperature using commercial etched silicon cantilevers with a tip of radius ranging from 5 to $10 \mathrm{~nm}$ and a spring constant of 20-100 $\mathrm{N} \mathrm{m}^{-1}$. Image analysis was performed using the NanoScope III software (version 512r2).

Immobilization of the nanoparticles on mica surfaces. To determine their mechanical properties, the NPs were grafted onto modified mica surfaces as described elsewhere. ${ }^{15}$ Briefly, large sheets $(1.5 \times 4 \mathrm{~cm})$ of freshly cleaved mica surfaces were first plasma treated to activate $\mathrm{OH}$ groups on their surface as described in detail elsewhere. ${ }^{16,17}$ Plasma activation was performed for $5 \mathrm{~min}$ at $40 \mathrm{~W}$ using argon and water vapors at a partial pressure of $80 \mathrm{mT}$ Torr and $300 \mathrm{mT}$ Torr respectively. After the plasma treatment, the mica surfaces were left in the plasma chamber under vacuum ( $0.5 \mathrm{mT}$ Torr $)$ for $5 \mathrm{~min}$. Then, the surfaces were transferred to an evaporation chamber and stored under vacuum $(1.6 \mathrm{mmHg})$. The evaporation chamber was connected via a valve to a small glass reservoir containing $100 \mu \mathrm{L}$ of APTES. After purging the evaporation chamber for $15 \mathrm{~min}$, the valve was opened allowing APTES vapors to react with the activated mica surfaces. Evaporation was allowed to proceed for $4 \mathrm{~h}$. The valve was closed and remnant APTES vapors were pumped out for $2 \mathrm{~h}$. The grafting reaction of APTES was completed by annealing the surfaces for $30 \mathrm{~min}$ at $90{ }^{\circ} \mathrm{C}$ under atmospheric pressure. Then, the surfaces bearing grafted APTES molecules were immersed overnight in a $1 \%(\mathrm{w} / \mathrm{w})$ water solution of glutaraldehyde where the coupling reaction between APTES amine function and glutaraldehyde carbonyl functional groups took place in presence of the catalytic agent $\mathrm{NaBH}_{3} \mathrm{CN}$. The resulting glutaraldehyde-functionalized mica surfaces were thoroughly rinsed with Milli-Q ${ }^{\circledR}$ water prior to NP deposition. Deposition was performed using the horizontal convective evaporation method. ${ }^{18}$ A small drop $(25 \mu \mathrm{L})$ of a NP suspension $(0.1 \% \mathrm{w} / \mathrm{w}$ in DMEM) was placed between a glass applicator and the functionalized mica surface prior to substrate translation. As the surface was translated at a fixed velocity, the NP suspension drop could spread on the substrate forming a uniform monolayer of nanoparticles. The final concentration of grafted NPs on the surfaces was $2.6 \pm 0.4 \mathrm{NPs} / \mu^{2}$ as determined by AFM imaging.

Mechanical properties of the nanoparticles. The mechanical properties of the NPs grafted on mica surfaces were measured in 
PBS at $37{ }^{\circ} \mathrm{C}$ using a Bioscope AFM equipped with a G-type piezotube scanner, Nanoscope IIIa controller, and version 4.43r8 of the Nanoscope software (Veeco Metrology, Santa Barbara, CA). Silicon nitride microlevers with spring constant $k=0.01 \mathrm{~N} \mathrm{~m}^{-1}$ were used. Mica substrates bearing grafted NPs were immersed in PBS at $37{ }^{\circ} \mathrm{C} 1 \mathrm{~h}$ prior to measurement. Localization of the NPs was realized by scanning the surface in contact mode over a $3 \times 3 \mu \mathrm{m}^{2}$ area. The scanning area was progressively decreased to visualize only one NP in the scanned area. Force profiles were measured by approaching the tip to the NP on the surface at a fixed velocity and recording the cantilever deflection. Young moduli were extracted from experimental curves by fitting the force profiles with the Hertz model for a conical indenter: ${ }^{19,20}$

$$
F=\frac{2 E \tan \theta}{\pi\left(1-\nu^{2}\right)} \delta^{2}
$$

where $F$ is the indentation force, $E$ and $\nu$ are the Young modulus and the Poisson ratio of the NPs respectively, $\delta$ is the indentation and $\theta$ is the half-opening angle of the indenter. One monolayer of NPs was prepared for each of the different NP batches. Five to ten force profiles were recorded on one single NP and six different NPs were analyzed for all surfaces. The Young moduli reported in this study represent the average value obtained from all recorded force profiles.

\section{Nanoparticle uptake by living cells}

Cell culture. Macrophage cells, RAW 264.7 were a kind gift from Professor J. C. Leroux (Université de Montréal). Cells were grown in complete medium consisting of Dulbecco's modified Eagle culture medium (DMEM) containing 10\% (v/v) heatinactivated fetal bovine serum, $100 \mathrm{U} \mathrm{mL}^{-1}$ penicillin-G, and $100 \mathrm{mg} \mathrm{mL}^{-1}$ streptomycin (Invitrogen, Burlington, ON, Canada) in a $5 \% \mathrm{CO}_{2}$ atmosphere at $37^{\circ} \mathrm{C}$. The cells were used between passage 4 and 15 .

Uptake study. RAW 264.7 cells were plated in 96-well flat bottom plates $\left(2 \times 10^{5}\right.$ cells/well $)$ and allowed to adhere overnight in complete medium. Then, the medium was removed and replaced with DMEM. Various volumes of a fluorescentlylabeled NP suspension $(0.1 \% \mathrm{w} / \mathrm{w}$ in DMEM) were added to the cells to obtain different NP concentrations. The cellular uptake of the NPs was allowed to occur for $2 \mathrm{~h}$ at $37{ }^{\circ} \mathrm{C}$. The cells were washed with PBS and lysed with $0.2 \%$ (w/v) Triton-X 100 in $0.2 \mathrm{~N} \mathrm{NaOH}$ solution. The lysate fluorescence was measured on a microplate reader at excitation and emission wavelengths of 550 and $580 \mathrm{~nm}$ respectively. The concentration of internalized NPs was determined from an internal calibration curve. This internal calibration curve, i.e. fluorescence intensity versus $\mathrm{NP}$ concentration, was established by measuring the fluorescence of a known NP concentration under the same conditions of cell incubation but without washing the cells with PBS. The uptake kinetics of the NPs were also investigated via fluorescence analysis. For a fixed NP concentration of $70 \mu \mathrm{g} \mathrm{mL} L^{-1}$, the cells were treated with the NPs at different incubation times and lysed as previously described.

To determine the uptake mechanism of the NPs, cells were incubated with endocytic and metabolic inhibitors prior to NP introduction. The cells were incubated in complete medium in the presence of one inhibitor, whose concentration is given in Fig. 3, for $1 \mathrm{~h}$ and then incubated with the NP solution $\left(70 \mu \mathrm{gL}^{-1}\right.$, serum free medium) for $2 \mathrm{~h}$. Cells were washed three times with PBS, lysed and the lysate fluorescence was measured.

All uptake experiments were repeated three times in 96-well plates from Corning (Costar, Corning, NY, USA).

The toxicity of the inhibitors at the concentrations used in the study was assessed by standard MTT assay. Results showed that no significant effect of cell viability could be observed of a period of time of $24 \mathrm{~h}$ (data not shown).

Intracellular trafficking. To establish the intracellular pathways pursued by the NPs, RAW 264.7 cells were plated on roundshape cover slips (Fisher Scientific, CA) and incubated overnight at $37{ }^{\circ} \mathrm{C}$ in the presence of fluorescein isothiocyanate-labeled dextran (DX-FITC, $M_{\mathrm{w}} 77000 \mathrm{~g} \mathrm{~mol}^{-1}, 1 \mathrm{mg} \mathrm{mL}^{-1}$ in complete medium) as lysosome marker. Then, the cells were washed with DMEM and $8 \mu \mathrm{L}$ of NP suspension were added to reach a final concentration of $70 \mu \mathrm{g} \mathrm{NPs} / \mathrm{mL}$ per well. After $20 \mathrm{~min}$ of incubation, the cells were washed with DMEM and incubated in complete medium for different incubation times allowing intracellular trafficking. Following incubation, the cells were fixed with a $4 \%(\mathrm{v} / \mathrm{v})$ formaldehyde solution for $10 \mathrm{~min}$ and mounted on glass slides for subsequent fluorescence microscopy analysis.

Staining of endocytic vesicles was done as follows. After fixation, the cells were incubated in $250 \mu \mathrm{L}$ of blocking solution ( $2 \% \mathrm{w} / \mathrm{v}$ bovine serum albumin in PBS) for $2 \mathrm{~h}$. Then the cells were incubated overnight at $4{ }^{\circ} \mathrm{C}$ in the presence of goat antimouse EEA1 (clone 281.7 from Santa Cruz Biotechnology, CA, USA) antibody at a concentration of $10 \mu \mathrm{g} \mathrm{mL}^{-1}$. After washing with PBS, anti-goat ALEXA FLUOR ${ }^{\circledR} 488$ (Invitrogen, Burlington, CA) secondary antibody was added for $2 \mathrm{~h}$.

All samples were analyzed on a fluorescence microscope (Olympus IX71 microscope, Olympus, Markham, ON) using filters optimized for the different fluorescent probes. Images were obtained using an Evolution VF camera (MediaCybernetics, Bethesda, MD) with a $100 \times$ objective lens and a constant optimal exposure time. All images were analyzed with ImagePro ${ }^{\circledR} 5.0$ (MediaCybernetics, Bethesda, MD) image analysis software.

All trafficking experiments were repeated three times in 24-well plates from Corning (Costar, Corning, NY, USA).

Statistical analysis of in vitro experiments. All in vitro experiments were repeated three times and values are presented as the mean \pm standard deviation. Statistical significance was determined by a Student's $t$-test.

\section{Results and discussion}

\section{Physical properties of the nanoparticles}

All NPs were synthesized by emulsion polymerization at a constant ratio of $9: 1$ of DEA:HEMA monomers. Four different batches were prepared using four different crosslinker concentrations ranging from 1.7 to $15 \mathrm{~mol} \%$ (Table 1). A typical AFM image of the particles deposited via horizontal convective assembly on a bare mica substrate at $25^{\circ} \mathrm{C}$ is illustrated in Fig. 1(a). All NPs exhibit a round shape and a smooth surface 
Table 1 Properties of the nanoparticles. All measurements were done in PBS at $37^{\circ} \mathrm{C}^{a}$

\begin{tabular}{lccrr}
\hline Batch name & Crosslinker content $(\mathrm{mol} /)$ & Particle size/nm & Zeta-potential/mV & Young modulus/kPa \\
\hline A & 1.7 & $169.9 \pm 13.3$ & $-8.0 \pm 1.0$ & $18.04 \pm 4.98$ \\
$\mathrm{~B}$ & 5 & $162.1 \pm 22.8$ & $-10.7 \pm 1.4$ & $35.84 \pm 10.84$ \\
$\mathrm{C}$ & 10 & $176.4 \pm 28.4$ & $-7.8 \pm 2.0$ & $136.28 \pm 39.55$ \\
$\mathrm{D}$ & 15 & $153.0 \pm 35.3$ & $-13.6 \pm 2.3$ & $211.39 \pm 43.28$ \\
${ }^{a}$ Values are presented as the mean \pm standard deviation. & & & \\
\hline
\end{tabular}

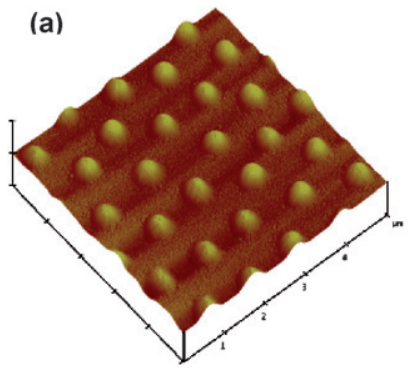

(b)

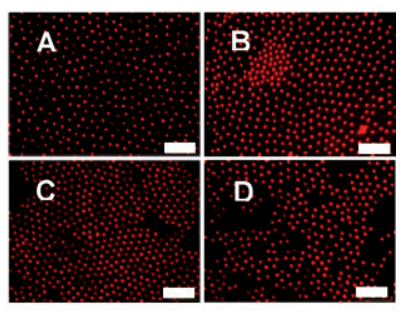

Fig. 1 Surface imaging of the hydrogel nanoparticles (a) deposited from PBS solution via horizontal convective assembly at $37{ }^{\circ} \mathrm{C}$ on a mica surface and analyzed using AFM in air at $25^{\circ} \mathrm{C}$ in tapping mode (see Experimental for details) (b) NPs deposited on glass slides by self-adsorption from PBS solution at $37{ }^{\circ} \mathrm{C}$ and analyzed using fluorescence microscopy. No particle aggregates were observed under the experimental conditions used. (a) Vertical scale bar $=100 \mathrm{~nm}$, (b) Scale bars $=5 \mu \mathrm{m}$.

topography. No aggregates were observed for all batches under the deposition conditions used. The NP sizes measured by dynamic light scattering in PBS at $37{ }^{\circ} \mathrm{C}$, which is well above their lower critical temperature, are reported in Table 1. The particle size is independent of the crosslinker concentration and ranges from 150 to $170 \mathrm{~nm}$. Zeta-potentials of the NPs at $37^{\circ} \mathrm{C}$ in PBS are also reported in Table 1 . The values are slightly negative for all NP batches. However, the variability in zeta-potential for such low potentials is known to be $c a . \pm 5 \mathrm{~V}$. Therefore, the NP surface potential is assumed to be negligible. NPs were also observed by fluorescence microscopy in order to assess their aggregation state under the experimental conditions used for in vitro experiments. A drop of NP suspension $(0.1 \% \mathrm{w} / \mathrm{w}$ in DMEM) was deposited on glass cover slips and mounted on slides for fluorescence microscopy analysis. Images shown in Fig. 1(b) reveal that the NPs are well organized on the substrate and do not form aggregates. These results suggest the stability, i.e. the good dispersion, of isolated NPs under the experimental conditions used.

The Young modulus of the NPs chemically grafted on functionalized mica surfaces were extracted from force profiles measured using AFM in PBS at $37^{\circ} \mathrm{C}$. Only force curves obtained on approach, as illustrated in Fig. 2, were used to determine Young moduli. The force curves were repeated several times on the same contact point of the particle. The results were reproducible and therefore confirm the absence of irreversible deformation of the NPs during the indentation. Young modulus of the NPs was calculated by fitting the experimental data to eqn (1) considering a Poisson ratio of 0.5 (rubber like material). As illustrated in Table 1, the increase in Young modulus from batch

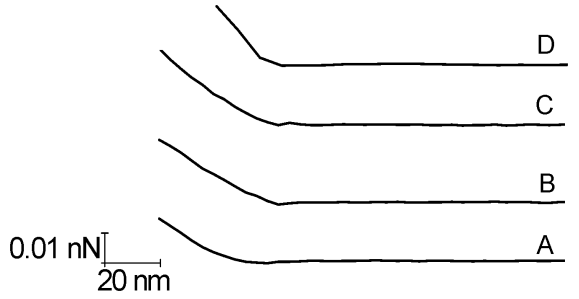

Fig. 2 Characteristic force curves measured using AFM between a cantilever tip and one nanoparticle grafted on a mica surface for the NP batches listed in Table 1 . The grafted NPs were immersed in PBS at $37^{\circ} \mathrm{C}$ for $1 \mathrm{~h}$ prior to the experiment. The increase in the slope of the curve in the indentation regime (corresponding to the left part of the force curve) from batch A to batch D corresponds to an increase in Young modulus as described by the eqn (1).

A to $\mathrm{D}$ is directly related to the increase in the crosslinker concentration as expected from elastic theory of rubber-like materials. $^{21}$ The Young moduli of the NPs from batch A (1.7 mol\% of crosslinker) and batch $\mathrm{D}$ (15 mol $\%$ of crosslinker) are $18.04 \pm 4.98$ and $211.39 \pm 43.28 \mathrm{kPa}$ respectively which are in good agreement with reported elastic moduli of NIPAM NPs. ${ }^{22,23}$

\section{Uptake mechanism and intracellular fate of the nanoparticles}

Uptake experiments are usually performed at $4{ }^{\circ} \mathrm{C}$ in order to stop any energy dependent process like endocytosis in the cell. However, these experiments could not be performed with our hydrogel NPs because they are thermosensitive. Indeed, a change in temperature induces a change in NPs size which could give rise to a change in their elastic properties. Therefore, the uptake mechanism of the NPs was investigated by treating the cells with endocytic and metabolic inhibitors prior to incubation with the NP solutions. Sodium azide was used at $37^{\circ} \mathrm{C}$ as a metabolic inhibitor as it prevents the production of ATP by interfering with the glycolytic and oxydative metabolic pathways of the cell. ${ }^{24,25}$ Fig. 3 illustrates the uptake concentration of NPs in sodium azide-treated cells normalized by the uptake by non-treated cells. Upon treatment, the intracellular concentration of NPs for the four different batches corresponds to $40 \%$ of the concentration found in non-treated cells indicating the presence of an active uptake process (Fig. 3).

Chlorpromazine was used for the specific inhibition of clathrin-mediated endocytosis as it is known to impede the formation and budding of clathrin-coated pits. ${ }^{26}$ When treated with chlorpromazine, the NP concentrations for batches B, C and D correspond to $50 \%$ of the concentration measured in the counterpart non-treated cells while it reaches $80 \%$ for batch $\mathrm{A}$. This means that NPs from batches B, C and D are internalized by 


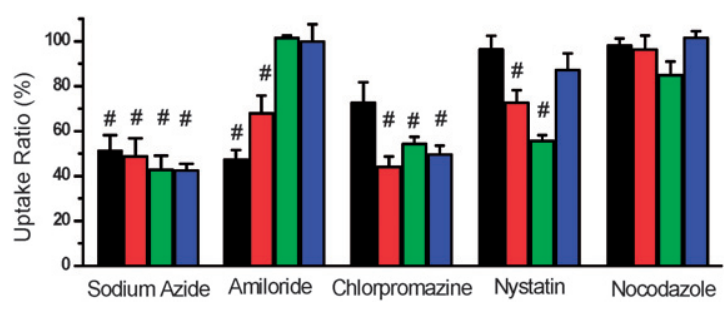

Fig. 3 Cellular uptake mechanisms of the NPs assessed by treating the cells with different inhibitors of endocytic entry routes. The drug concentrations used were: Sodium azide: $100 \mathrm{mM}$, amiloride: $200 \mu \mathrm{g}$ $\mathrm{mL}^{-1}$, chlorpromazine: $200 \mu \mathrm{g} \mathrm{mL}^{-1}$, nystatin: $100 \mathrm{U} \mathrm{mL}^{-1}$, nocodazole: $5 \mu \mathrm{g} \mathrm{mL}{ }^{-1}$. Cells were incubated with the drug $1 \mathrm{~h}$ prior to NP treatment (see Experimental for details). After $2 \mathrm{~h}$ of NP treatment, cells were washed, lysed and lysate fluorescence was quantified. The uptake ratio represents the fluorescence intensity in the presence of added drug normalized by the fluorescence intensity under control conditions (without added drug) Black: batch A; red: batch B; green: batch C; blue: batch D. (\#: data significantly inferior to $100 \%, n=3, p<0.001$ ).

clathrin mediated endocytosis but not the NPs from batch A (Fig. 3).

Nystatin was used to inhibit caveolae-mediated endocytosis. This drug is well known to perturb cholesterol-rich membrane microdomains which are precursors of caveolae invaginations. ${ }^{24}$ The cellular uptake of NPs from the batches A and D was not significantly affected by the presence of the drug, indicating that none of these particles are taken up by caveolae-mediated endocytosis (Fig. 3). Uptake concentrations for batches B and C were significantly reduced (30\% and $45 \%$ of the concentration found in non-treated cells) in the presence of nystatin which confirms that the internalization mechanism of the NPs is partially caveolae dependent.

Amiloride is an inhibitor of the $\mathrm{Na}^{+} / \mathrm{H}^{+}$exchange protein and was used to hinder macropinocytosis. ${ }^{27}$ The cellular uptake of NPs in the presence of amiloride for batches A and B correspond to $45 \%$ and $65 \%$ of the concentration found in non-treated cells respectively indicating that cellular uptake is mediated by macropinocytosis. No effect of amiloride was observed for NPs from batches $\mathrm{C}$ and $\mathrm{D}$ suggesting the absence of macropinocytosis for the internalization of these NPs (Fig. 3).

Nocodazole is known to inhibit microtubule assembly which is involved in the intracellular trafficking of vesicles. ${ }^{28}$ No significant effect of nocodazole treatment on cell uptake was observed for all NP batches indicating that the uptake is microtubule independent (Fig. 3).
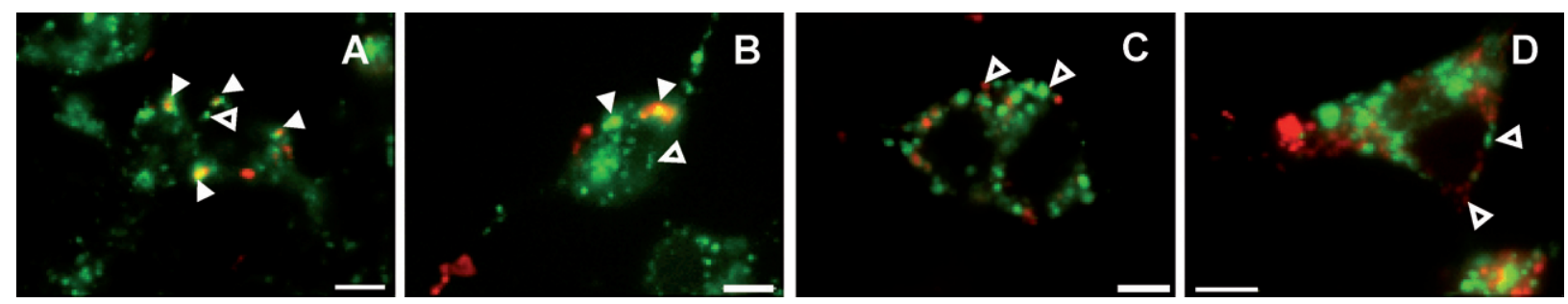

Fig. 4 Fluorescence micrographs of macrophage cells incubated simultaneously for $1 \mathrm{~h}$ with DX-FITC (green channel) and NPs (red channel) from batch A to D. After abundant rinsing, cells were incubated with complete medium during 15 min chase. The internalization of DX-FITC and NPs in small spherical vesicles is indicated by the empty arrows. Colocalization of both species is observed for NPs from batches A and B (filled arrows, images A and B) while segregation for NPs from batches C and D is observed (images C and D). Scale bar $=5 \mu \mathrm{m}$. 


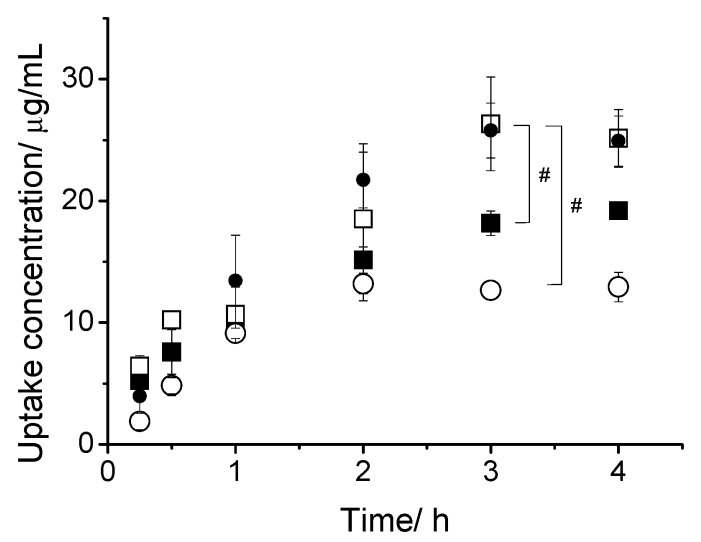

Fig. 6 Uptake kinetics of NPs by macrophage cells. Cells were treated with a $70 \mu \mathrm{g} \mathrm{mL}^{-1} \mathrm{NP}$ suspension for a certain period of time. After rinsing with PBS, cells were lysed and fluorescence was quantified by spectrophotometry. Results were converted to uptake concentrations using internal calibration. $\mathrm{O}$ : batch $\mathrm{A} ; \square$ : batch $\mathrm{B}$; $\bigcirc$ : batch $\mathrm{C}$; batch D. $(n=3, \#: p<0.001)$.

respectively). No signs of macrophage activation such as changes in cell morphology were observed during the experiments.

The kinetics of the cellular uptake were studied at $37^{\circ} \mathrm{C}$ for a NP concentration of $70 \mu \mathrm{g} \mathrm{mL} L^{-1}$. For all NP batches, the uptake concentration increases with time and reaches a plateau at $2.5 \pm 0.5 \mathrm{~h}$ (Fig. 6). The initial uptake kinetics are similar for all NP batches but the concentrations observed at the plateau are significantly different. Batches $\mathrm{B}$ and $\mathrm{C}$ exhibit large uptake concentrations $\left(25.1 \mu \mathrm{g} \mathrm{mL} L^{-1}\right.$ and $24.9 \mu \mathrm{g} \mathrm{mL}^{-1}$ respectively) compared to batch $\mathrm{A}\left(12.1 \mu \mathrm{g} \mathrm{mL} \mathrm{L}^{-1}\right)$ and batch $\mathrm{D}\left(12.9 \mu \mathrm{g} \mathrm{mL}^{-1}\right)$.

NP intracellular trafficking was observed for different chasing times using fluorescence microscopy. As shown in Fig. 7(a), the colocalization percentage of the NPs with DX-FITC increases with chasing time, for all NP batches, and reaches a plateau after a few hours. These results indicate that the compartments containing the internalized NPs were slowly merging with lysosomes and that NPs were not released in the cytosol during their transport. However, noticeable differences in the kinetics of intracellular trafficking were observed between the different NP batches. The NPs from batches B and C reached the lysosomes within $3 \mathrm{~h}$ and the NPs from batches A and D attained the lysosomes after $6 \mathrm{~h}$.

Fig. 7(b) shows the localization of the NPs and cell lysosomes after internalization. The NPs (red channel) are found to be colocalized with DX-FITC (green channel) after $3 \mathrm{~h}$ of chasing. After $6 \mathrm{~h}$ of chasing, NPs from all batches concentrated in large vesicles where DX-FITC is present at the periphery.

Colocalization of NPs with early endosome marker EEA-1 was difficult to assess. The main reason was the rapid trafficking of the vesicles in the cells upon NP internalization as already reported in human hepatocarcinoma cells. ${ }^{29}$ However, it was possible to detect some colocalization spots after $15 \mathrm{~min}$ chase exclusively in cells treated with batch D (Fig. 8). No colocalization of NPs with endosome markers was observed after $30 \mathrm{~min}$ chase for all NP batches.

The differences observed in the uptake mechanisms and kinetics are associated with the differences in the mechanical properties of the nanoparticles, as the Young modulus is the only characteristic parameter differentiating the four NP batches. (a)

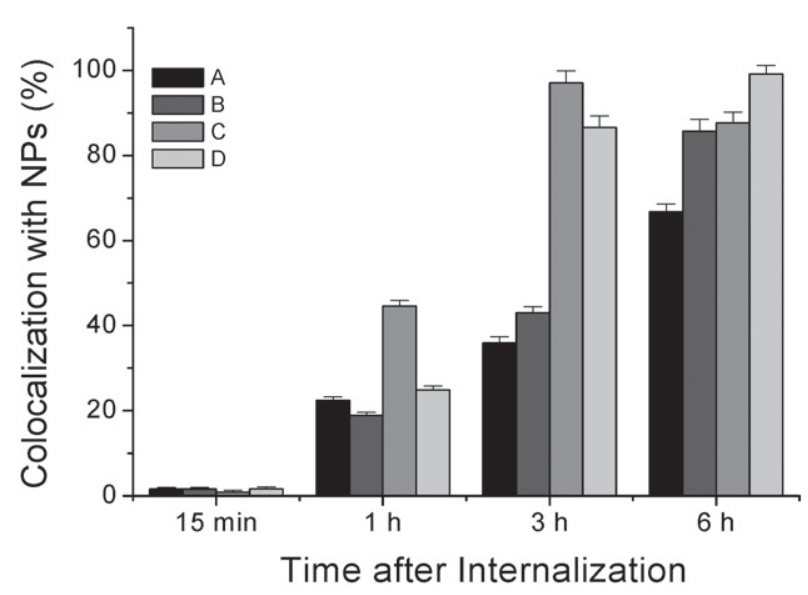

(b)

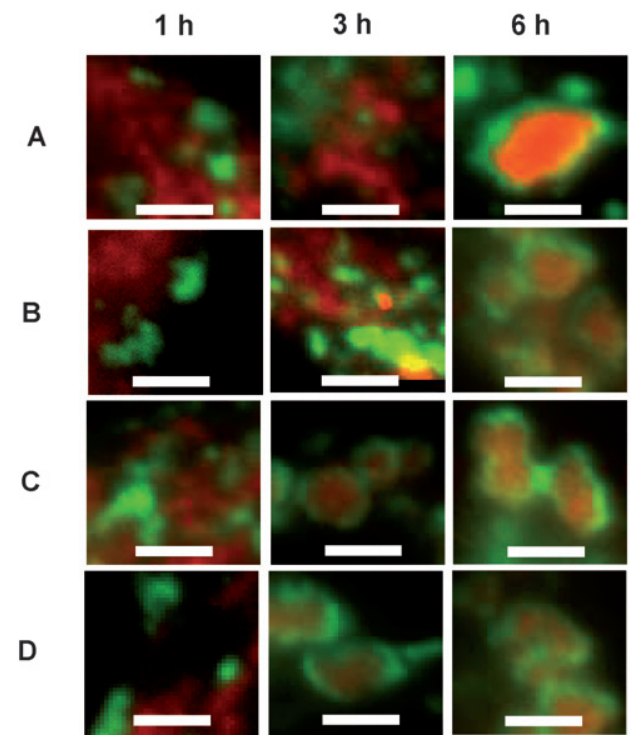

Fig. 7 (a) Percentage of colocalization of DX-FITC to rhodamine B-labeled NPs, quantified by fluorescence image analysis, as a function of time after internalization of the NPs. (b) Fluorescence micrographs of rhodamine B-labeled NPs in RAW 264.7 cells at different times after internalization. Cells were treated with green-marker DX-FITC $12 \mathrm{~h}$ prior to the NP treatment in order to label cell lysosomes. Scale bar $=2 \mu \mathrm{m}$.

Results show an inhibition of NP uptake in the presence of sodium azide for all NP batches suggesting the presence of an active process of internalization. Nanoparticles with low Young modulus are almost exclusively internalized by macropinocytosis while more elastic nanoparticles (batch $\mathrm{C}$ and $\mathrm{D}$ ) exhibit clathrin-and/or caveolae-mediated entry routes. The highly elastic NPs (batch D) are taken up mainly by clathrin-mediated endocytosis. Previous studies have shown that the physical structure of macromolecules plays a role in determining their cellular uptake mechanism. Ma et al. ${ }^{30}$ recently showed that free chitosan macromolecules were internalized by Caco-2 cells via caveolaemediated endocytosis while NPs made of crosslinked chitosan were internalized via clathrin-mediated endocytosis. Chitosan NPs showed larger uptake concentrations compared to free chitosan. Differences in binding and cellular uptake between 


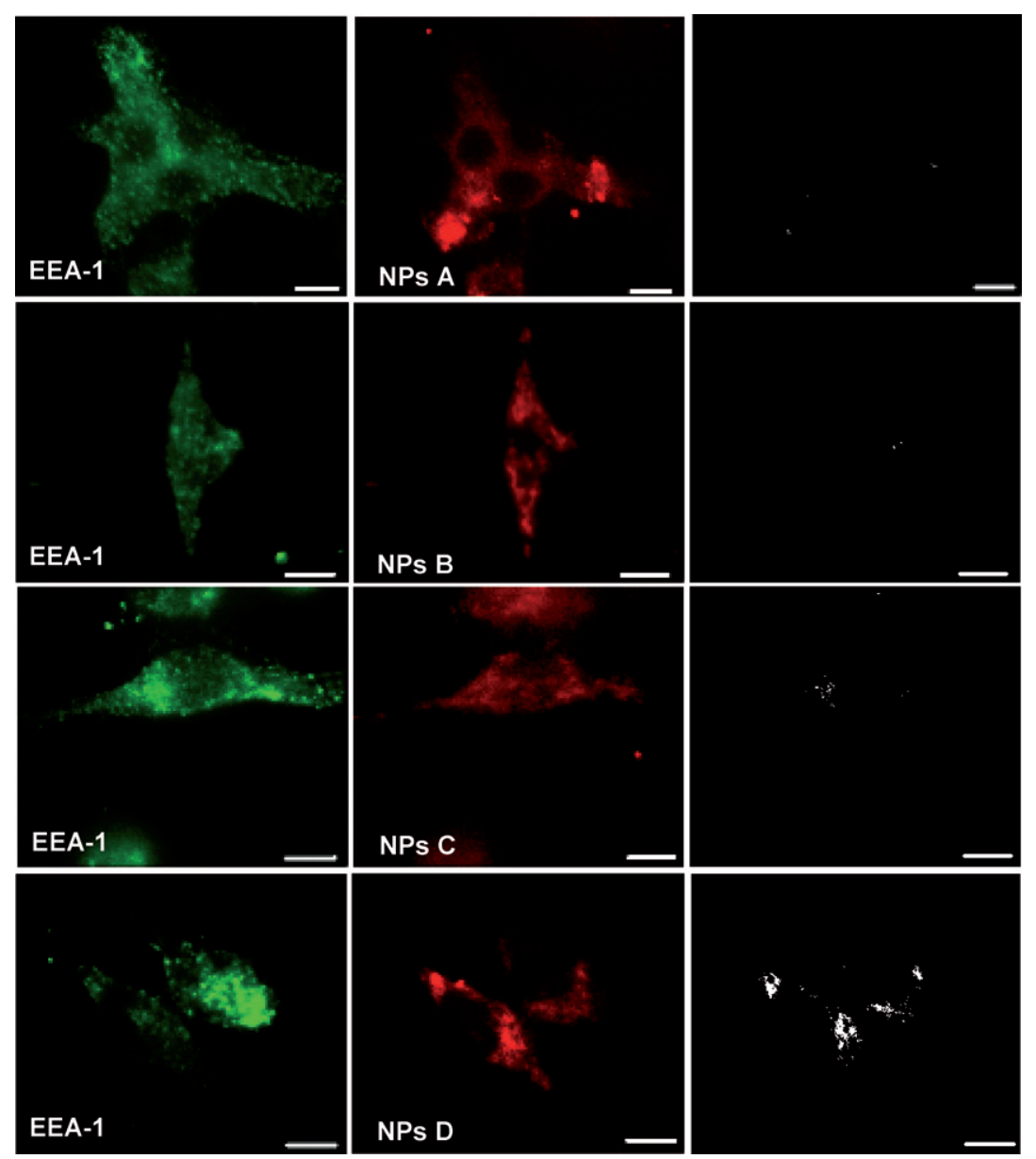

Fig. 8 Fluorescence micrographs showing colocalization of NPs (red channel) with early endosome marker EEA-1 (green channel). Cells were incubated with NPs for $1 \mathrm{~h}$ and chased for $15 \mathrm{~min}$. Cells were washed and fixed. Endosome staining was performed using goat anti-mouse EEA-1 mAb followed by a secondary antibody (Alexa 488) to reveal vesicle localization. The colocalization mask of the red and green channels is represented in white. From these micrographs, only NPs from batch D exhibit significant colocalization. Scale bar $=5 \mu \mathrm{m}$.

linear and branched polyethylene imines (PEI) have also been observed. ${ }^{31}$ Branched PEI demonstrated five -fold greater binding to B16f10 melanoma cells than its linear homologue. Addition of methyl- $b$-cyclodextrin which sequesters cholesterolinhibiting caveolae-mediated endocytosis induced a dramatic decrease in the cell uptake of branched PEI while it increased the uptake of linear PEI. Although the different mechanisms observed were not directly correlated with the differences in particle size or elasticity, these reported studies also show that the physical structure of carriers affects cellular uptake mechanisms.

Differences in the NP uptake concentration are also observed between the different NP batches. NPs with intermediate Young modulus (batch $\mathrm{B}$ and $\mathrm{C}$ ) give rise to relatively large uptake concentrations because they exhibit multiple entry mechanisms. In comparison, the NPs from batch A and D, which are internalized only by one mechanism, exhibit smaller uptake concentrations.

Significant contribution of macropinocytosis in the entry routes for batch $\mathrm{A}$ and $\mathrm{B}$ was confirmed by fluorescence microscopy. Cells treated simultaneously with DX-FITC and NPs present small spherical vesicles containing both NPs and dextran after $15 \mathrm{~min}$ of chase. As DX-FITC is well known to be internalized via macropinocytosis and phagocytosis, ${ }^{32}$ the existence of a significant number of NPs close to DX-FITC for the batches $\mathrm{A}$ and $\mathrm{B}$ indicates a significant contribution of macropinocytosis for these batches compared to batches $\mathrm{C}$ and D. Macropinocytosis and clathrin-mediated endocytosis are two mechanisms by which internalized particles can traffic to lysosomal compartments, whereas caveolae-mediated endocytosis does not systematically give rise to accumulation in lysosomal compartments. ${ }^{33}$ Because all NP batches are found to be partly or mainly internalized via macropinocytosis and/or clathrinmediated endocytosis (Fig. 3), they all converged to lysosomal compartments independently of their elasticity.

Colocalization of NPs with the endosomal marker EEA-1 reveals that NPs from batch $\mathrm{A}$ to $\mathrm{C}$ are not internalized via endosomal vesicles, which is consistent with a non-clathrinmediated entry mechanism. Colocalization of NPs from batch D with an early endosomal marker after $15 \mathrm{~min}$ chase, indicates that internalized NPs are rapidly delivered to sorting vesicles. Addition of nocodazole, which inhibits microtubule-dependent transport, has been reported to stop the transport cycle from sorting to recycling vesicles. ${ }^{29}$ No effect of nocodazole was observed on the uptake of NPs from all batches. Therefore, a very low level of partitioning of NPs from early endosomes to recycling vesicles was expected to occur. This is also supported by the fact that all NP batches exhibited similar levels of 
colocalization with lysosomal marker DX-FITC after $6 \mathrm{~h}$ chase. It is noteworthy that microtubule-independent transport mechanism to lysosomes discards the possibility of uptake by phagocytosis. ${ }^{34,35}$

The particle size can strongly affect the uptake mechanism. Recently, it has been reported that particles of diameter lower than $200 \mathrm{~nm}$ were preferentially internalized via clathrin-coated pits, whereas particles of $500 \mathrm{~nm}$ diameter penetrated into the cells via the caveolae. ${ }^{11}$ The multiplicity of entry mechanism observed in our study for batches B and C can not be attributed to particle size or aggregation of particles because the particle size is not significatly different between the different NP batches and no signs of aggregation induced by the high salinity of the medium were evident during fluorescence microscopy. NP aggregation in vitro was observed only after 2 to $3 \mathrm{~h}$ of chase. Our results clearly indicate that the cellular uptake mechanism and kinetics of the NPs are also regulated by the particle elasticity.

The mechanism by which NP elasticity modulates the entry route remains unknown. However, the dynamics of the membrane-NP interface deformation is most probably playing a role in controlling the cellular uptake. As a NP approaches the cell membrane, the thermal fluctuations of the membrane-NP interface could impede the formation of stable contacts and subsequent receptor recruitment. As soft NPs are expected to deform more easily under thermal fluctuation forces than the more elastic NPs, their binding with the cell membrane could be less favorable. Therefore, soft NP uptake would be expected to be mediated by a receptor-independent mechanism such as macropinocytosis, while more elastic NPs could trigger receptordependent internalization. This hypothesis is well supported by our experimental data but also by studies using hard nanoparticles. Indeed, the internalization of hard polymeric or non-polymeric nanoparticles has been reported to be clathrindependent in the vast majority of cases. ${ }^{36}$

\section{Conclusions}

We investigated the relationship between the mechanical properties of hydrogel nanoparticles (NPs) assessed by the Young modulus, and their cellular uptake mechanisms and kinetics. All NPs were internalized by an energy-dependent mechanism but the endocytic mechanism was shown to be dependant on the NP elasticity. Soft NPs are preferentially internalized by macropinocytosis while hard sphere uptake involves clathrin-mediated routes. NPs exhibiting intermediate elasticity are found to be internalized via multiple mechanisms which gives rise to a larger uptake rate.

The present study confirmed that the elasticity of hydrogel NPs influences their cellular entry routes. The control of mechanical properties of hydrogel NPs represents a promising route to the design of new drug carriers with specific subcellular delivery.

\section{Acknowledgements}

This research was funded by the Groupe de Recherche Universitaire de l'Université de Montréal and the Natural Sciences and Engineering Research Council of Canada. A. A. is supported by the E. A. Baker Foundation and The Institute of
Neuroscience, Mental Health and Addiction (INMHA) of the Canadian Institutes of Health Research (CIHR). F. S. is supported by CONACYT. J-F. B. is supported by a Health Research Foundation R\&D Canadian Institutes of Health Research Scholarship. The authors thank Professor R. E. Prud'homme for providing access to his AFM facilities.

\section{References}

1 J. O. You and D. T. Auguste, Biomaterials, 2008, 29, 1950.

2 K. T. Nguyen, K. P. Shukla, M. Moctezuma, A. R. C. Braden, J. Z. Zhibing and H. L. Tang, J. Biomed. Mater. Res., Part A, 2008, 88A, 1020

3 Y. Li, G. Sun, J. Xu and K. L. Wooley, in Nanotechnology in Therapeutics: Current technology and applications, ed. J. Z. Hilt, Horizon Bioscience, Wymondham, UK, 2007, pp. 381-407.

4 J. L. Turner, Z. Y. Chen and K. L. Wooley, J. Controlled Release, 2005, 109, 189.

5 O. P. Perumal, R. Inapagolla, S. Kannan and R. M. Kannan, Biomaterials, 2008, 29, 3469.

6 H. A. Clark, M. Hoyer, M. A. Philbert and R. Kopelman, Anal. Chem., 1999, 71, 4831.

7 H. A. Clark, R. Kopelman, R. Tjalkens and M. A. Philbert, Anal. Chem., 1999, 71, 4837.

8 H. Xu, J. W. Aylott, R. Kopelman, T. J. Miller and M. A. Philbert, Anal. Chem., 2001, 73, 4124.

9 I. A. Khalil, K. Kogure, H. Akita and H. Harashima, Pharmacol. Rev., 2006, 58, 32.

10 O. Harush-Frenkel, E. Rozentur, S. Benita and Y. Altschuler, Biomacromolecules, 2008, 9, 435.

11 J. Rejman, V. Oberle, I. S. Zuhorn and D. Hoekstra, Biochem. J., 2004, 377, 159.

12 S. K. Lai, K. Hida, S. T. Man, C. Chen, C. Machamer, T. A. Schroer and J. Hanes, Biomaterials, 2007, 28, 2876.

13 H. J. Kong, J. D. Liu, K. Riddle, T. Matsumoto, K. Leach and D. J. Mooney, Nat. Mater., 2005, 4, 460.

14 M. Colonne, Y. Chen, K. Wu, S. Freiberg, S. Giasson and X. X. Zhu, Bioconjugate Chem., 2007, 18, 999.

15 X. Banquy, X. X. Zhu and S. Giasson, J. Phys. Chem. B, 2008, 112, 12208.

16 B. Liberelle, X. Banquy and S. Giasson, Langmuir, 2008, 24, 3280.

17 B. Liberelle and S. Giasson, Langmuir, 2007, 23, 9263.

18 B. G. Prevo and O. D. Velev, Langmuir, 2004, 20, 2099.

19 K. L. Johnson, Contact mechanics, Cambridge University Press, Cambridge, 1st edn, 1987.

20 M. Radmacher, M. Fritz and P. K. Hansma, Biophys. J., 1995, 69, 264.

21 P. J. Flory, Polymer, 1979, $20,1317$.

22 O. Tagit, N. Tomczak and G. J. Vancso, Small, 2008, 4, 119.

23 J. Wiedemair, M. J. Serpe, J. Kim, J. F. Masson, L. A. Lyon, B. Mizaikoff and C. Kranz, Langmuir, 2007, 23, 130.

24 C. Lamaze and S. L. Schmid, Curr. Opin. Cell Biol., 1995, 7, 573.

25 J. Saraste, G. E. Palade and M. G. Farquhar, Proc. Natl. Acad. Sci. U. S. A., 1986, 83, 6425.

26 L. H. Wang, K. G. Rothberg and R. G. W. Anderson, J. Cell Biol., 1993, 123, 1107

27 L. J. Hewlett, A. R. Prescott and C. Watts, J. Cell Biol., 1994, 124, 689.

28 G. W. Zieve, D. Turnbull, J. M. Mullins and J. R. McIntosh, Exp. Cell Res., 1980, 126, 397.

29 C. Goncalves, E. Mennesson, R. Fuchs, J. P. Gorvel, P. Midoux and C. Pichon, Mol. Ther., 2004, 10, 373.

30 Z. S. Ma and L. Y. Lim, Pharm. Res., 2003, 20, 1812.

31 F. P. Seib, A. T. Jones and R. Duncan, J. Controlled Release, 2007, 117, 291.

32 U. Hacker, R. Albrecht and M. Maniak, J. Cell Sci., 1997, 110, 105.

33 A. Ferrari, V. Pellegrini, C. Arcangeli, A. Fittipaldi, M. Giacca and F. Beltram, Mol. Ther., 2003, 8, 284.

34 S. D. Conner and S. L. Schmid, Nature, 2003, 422, 37.

35 L. A. H. Allen and A. Aderem, Curr. Opin. Immunol., 1996, 8, 36.

36 O. Harush-Frenkel, Y. Altschuler and S. Benita, Crit. Rev. Ther. Drug Carrier Syst., 2008, 25, 485. 